\title{
PHOTOEMISSION MEASUREMENT ON NaCl IN THE PHOTON ENERGY RANGE 32-50 eV
}

\author{
R. Haensel, G. Keitel, G. Peters, P. Schreiber, and B. Sonntag \\ Physikalisches Staatsinstitut, II. Institut für Experimentalphysik der Universität Hamburg, Hamburg, Germany \\ and \\ C. Kunz* \\ Deutsches Elektronen-Synchrotron, Hamburg, Germany \\ (Received 18 July 1969)

\begin{abstract}
Photoemission measurements with photon energies ranging from the onset of $\mathrm{Na} 2 p$ electron absorption to $50 \mathrm{eV}$ have been performed using synchrotron radiation as a light source. The results indicate that the rise in the absorption coefficient starting at about $8.5 \mathrm{eV}$ above the onset of $2 p$ electron transitions is due to continuum transitions, whereas in the region below, most of the structures turn out to be exciton excitations.
\end{abstract}

Absorption measurements ${ }^{1,2}$ on the $\mathrm{Na}^{+} 2 p$ coreelectron excitations in $\mathrm{NaCl}$ have shown a rise in the absorption coefficient $\mu$ at about $8.5 \mathrm{eV}$ above the onset. The fundamental band-gap energy $E_{g}$ is approximately $8.5 \mathrm{eV}$. Most of the other $\mathrm{Na}$ halides as well as the alkali chlorides near the $\mathrm{Cl}^{-} 2 p$ onset have shown a similar behavior. Sagawa et al. ${ }^{2,3}$ and Iguchi et al. ${ }^{4}$ have attributed this rise in $\mu$ to two-electron excitations coming from the valence band and the core bands. Theoretical estimations made by Miyakawa ${ }^{5}$ yield a cross section for simultaneous two-electron excitations having a magnitude similar to one-electron excitations. On the other hand, Hermanson ${ }^{6}$ has estimated a probability for such a process which is smaller by two orders of magnitude, and we have recently been able to prove this experimentally for solid $\mathrm{Kr}$ and $\mathrm{Xe}^{7}$

We have made another experimental approach to this problem by measuring the photoemission of $\mathrm{NaCl}$. Our sample was mounted behind the exit slit of a Rowland monochromator ${ }^{8}$ and illuminated at normal incidence. The Deutsches Elektronen-Synchrotron 7.5-GeV synchrotron served as light source; the resolution was $0.1 \mathrm{eV}$. The sample ( 500- $\AA$ NaCl evaporated on gold) was connected to a sensitive dc amplifier (Cary Model No. 401) and was surrounded by a gold-plated cylindrical cup. A retarding voltage $U_{R}$ was applied between sample and cup. The spectral distribution of the photocurrent was recorded at different retarding voltages $U_{R}$. The measurements were repeated in a plane-grating spectrograph with less resolution but with essentially the same results.

First we would like to discuss the different types of processes leading to the emission of photoelectrons in our energy region and the particulars one has to know for interpreting our data.
(1) Though the intensity distribution at the exit slit is not accurately known, it has been verified that the spectrum is continuous and structureless. There is a monotonic increase of intensity to higher photon energies. Normal-incidence reflection of $\mathrm{NaCl}$ can be neglected.

(2) Figure 1 gives an outline of the relative energy positions of the conduction band $C D$, the valence band $V$, the core bands $C O$, and exciton levels $E X$ in $\mathrm{NaCl}$ and the Fermi levels $E_{\mathrm{F}}$, vacuum levels $V K$, and work functions $\Phi_{\mathrm{N}}$ and $\Phi_{G}$ in $\mathrm{NaCl}$ and gold. The external voltage $U_{R}$ is applied between the sample and the cup. We present below values for the different parameters, some of which are known only with an accuracy of $\pm 1 \mathrm{eV}$ which, however, is still good enough for the interpretation of our results. The bottom of the conduction band is $8.5 \mathrm{eV}$ above the top of the valence band ${ }^{9}$ and $35 \mathrm{eV}$ above the $2 p$ core bands

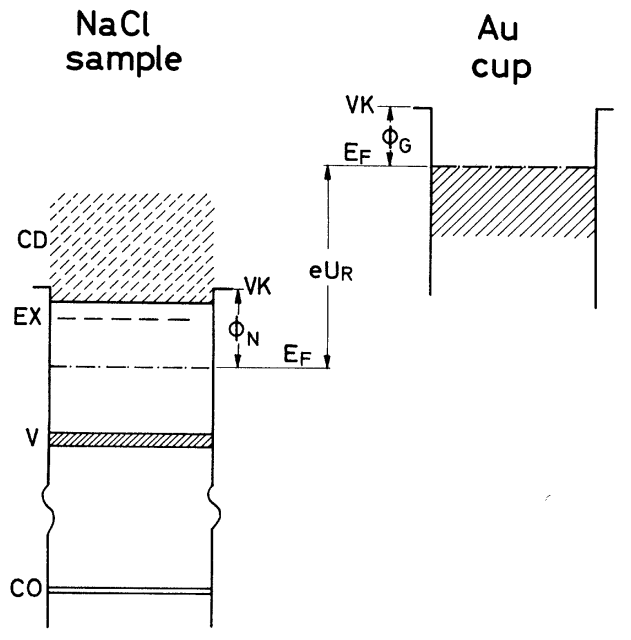

FIG. 1. Schematic drawing of the energy levels in a $\mathrm{NaCl}$ sample and gold-covered cup which was connected to the retarding potential $U_{R}$. Symbols are explained in the text [point (2)]. 
(assuming that the binding energy for the excitons in the absorption measurements is $2 \mathrm{eV}$ ). The work function of gold is $\Phi_{G} \approx 4 \mathrm{eV}$, and since it turned out experimentally that we obtained saturation of the photocurrent at $U_{R} \approx 0 \mathrm{~V}$, then it follows that $\Phi_{\mathrm{N}} \approx \Phi_{\mathrm{G}} \approx 4 \mathrm{eV}$. The vacuum level of $\mathrm{NaCl}$ coincides approximately with the bottom of the conduction band. This can be concluded from a measurement of the highest electron energy occurring at a fixed photon energy. Therefore $e U_{R}$ measures the kinetic energy in vacuum and is almost equal to the energy above the bottom of the conduction band. All this implies that the Fermi surface is - as is to be expected for pure samples - in the middle of the band gap of $\mathrm{NaCl}$; this is quite a surprising result for an evaporated film.

(3) Electrons excited from a core level into continuum states which suffer no energy loss before they escape from the sample are just rejected at a retarding potential $e U_{R}=-E_{c o}+\hbar \nu$. $E_{c 0}$ is the energy separation between the core state and the bottom of the conduction band ( 35 $\mathrm{eV})$. In the spectra this will give rise to a step at photon energies

$$
\hbar \nu=E_{c 0}+e U_{R} \text {. }
$$

This step can be distinguished from other structures since by changing $U_{R}$ the step changes its position in the spectra.

(4) Electrons with kinetic energies exceeding the band-gap energy $E_{g}(\sim 8.5 \mathrm{eV})$ can suffer energy loss due to electron-electron scattering.

(5) Excitons with a core-state hole can decay into high-energy photoelectrons (25 eV and more) due to ionization of a valence electron.

(6) Metastable excitons can dissociate into those continuum states which are at the same level.

(7) Core-state holes can make Auger transitions into the valence band thus emitting energetic electrons $(\sim 18 \mathrm{eV})$.

Figure 2 gives the spectral distribution of photoelectrons with regarding potential $U_{R}$ as parameter. The error in these curves is approximately $\pm 3 \%$ for $3 \times 10^{-13} \mathrm{~A}, \pm 5 \%$ for $3 \times 10^{-14} \mathrm{~A}$, and $\pm 15 \%$ for $3 \times 10^{-15} \mathrm{~A}$. For comparison we have added our earlier absorption measurement ${ }^{1}$ showing the rise in absorption from peaks $F$ to $G$ which Sagawa et al. attributed to two-electron excitations.

(a) Peaks $A$ to $F$ are not cut away by a step at a photon energy [given by Eq. (1)] which would otherwise identify them as continuum transitions according to point (3) or excitons decaying accord-

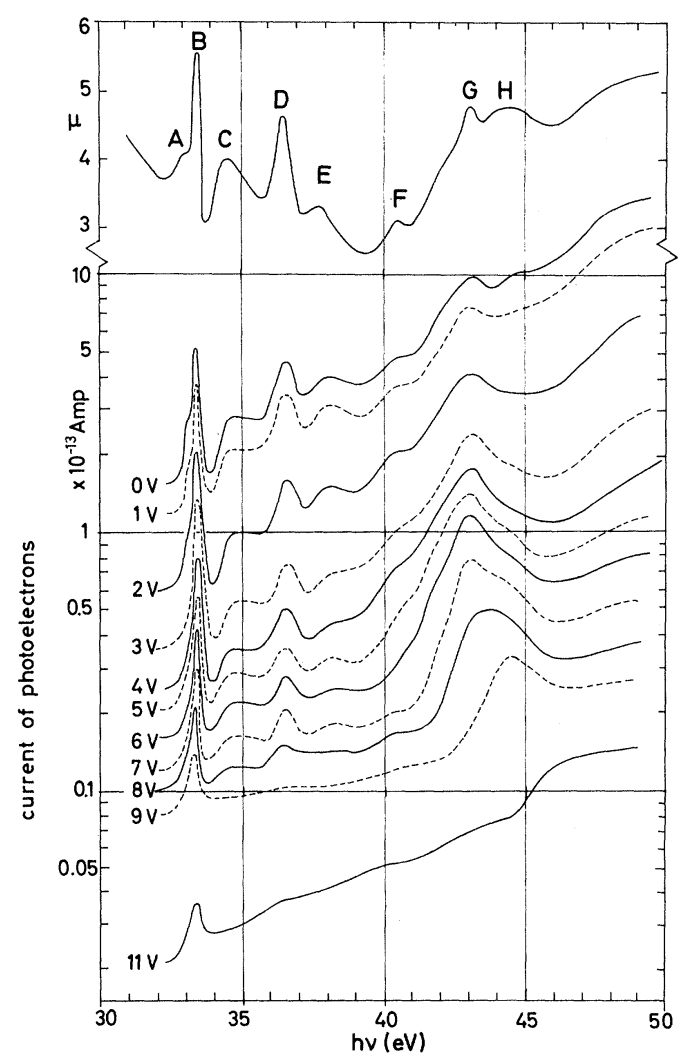

FIG. 2. Absorption coefficient $\mu$ in arbitrary units and currents of photoelectrons in logarithmic scale versus photon energy near the Na $L_{\mathrm{II}, \text { III }}$ edge in NaCl. The parameter of the different curves is the retarding potential $U_{R}$.

ing to point (6), but they continuously fade away with increasing retarding potential $e U_{R}$. We as sume that they are excitons decaying into photoelectrons according to point (5). [An experimental reason why Auger decay according to point (7) is negligible is given in section (b) below.] The photoelectrons with originally $25 \mathrm{eV}$ and more kinetic energy for peaks $A$ to $F$ will undergo energy losses according to point (4). Therefore a whole spectrum of electron energies is emitted. The peaks are preserved up to fairly high retarding potentials, finally merging into the background at values of $U_{R}$ depending on their intensity and the noise of the detecting system.

(b) In the spectrum with retarding voltage $U_{R}$ $=6 \mathrm{~V}$ a step of the type discussed in point (3) is found at photon energy $h \nu \approx 41 \mathrm{eV}$. This step reaches peak $G(\approx 43 \mathrm{eV})$ at $U_{R} \approx 8 \mathrm{~V}$. For $U_{R} \geqslant 9$ $\mathrm{V}$ peak $G$ is completely cut away. Therefore we conclude that $G$ is no double excitation but a continuum transition [an exciton excitation decaying according to point (6) cannot be excluded]。As with retarding voltage $U_{R} \geqslant 9 \mathrm{~V}$, no structure is 
left at position $G$, we conclude that Auger decay of the hole [see point (7)] is a negligible effect.

(c) Above peak $G$, electron transitions into oneelectron continuum states have kinetic energies high enough to suffer energy loss according to point (4); therefore many low-energy electrons are ejected into this region of the spectrum as may be seen from the different shapes of the spectra at $U_{R}=0 \mathrm{~V}$ and $U_{R} \geqslant 2 \mathrm{~V}$. But also electrons with their original energies are left, as can be seen from the step at $U_{R}=11 \mathrm{~V}$ according to point (3).

(d) At $U_{R}=11 \mathrm{~V}$, mainly a background of electrons ejected from the valence band is left for $h \nu$ $<45 \mathrm{eV}$. This reflects the intensity distribution behind the exit slit according to point (1). The only structure left is the strong exciton $B$ superimposed on this background.

We have given one possible consistent interpretation of our results which appears to us to be the most plausible within the frame of present knowledge on band structure and excitons。Of course, we are aware that this has to be tested by extending photoemission experiments to higher retarding voltages and other substances.

We thank the directors of the Deutsches Elektronen-Synchrotron and the Physikalisches Staatsinstitut, especially Professor P. Stähelin, for their interest in this work and for the generous support of the synchrotron radiation group. We are indebted to $\mathrm{Mr}$. Blechschmidt for having put a part of his equipment at our disposal, and we would also like to thank Dr. Skibowski for valuable discussions. Furthermore thanks are due to the Deutsche Forschungsgemeinschaft for financial support.

$\dagger$ Present address: Department of Physics and Astronomy, University of Maryland, College Park, Md. 20742

${ }^{1}$ R. Haensel, C. Kunz, T. Sasaki, and B. Sonntag, Phys. Rev. Letters 20, 1436 (1968).

${ }^{2} \mathrm{~T}$. Sagawa, in Soft X-Ray Band Spectra and the Electronic Structure of Metals and Material, edited by

D. J. Fabian (Academic Press, Inc., New York, 1968), p. 29 .

${ }^{3}$ T. Sagawa et al., J. Phys. Soc. Japan 21,2587 (1966).

${ }^{4}$ Y. Iguchi et al., Solid State Commun. $\underline{6}, 575$ (1968).

${ }^{5}$ T. Miyakawa, J. Phys. Soc. Japan 17, 1898 (1962).

${ }^{6} \mathrm{~J}$. C. Hermanson, Phys. Rev. 177, 1234 (1969).

${ }^{7}$ R. Haensel, G. Keitel, P. Schreiber, and C. Kunz, to be published.

${ }^{8}$ R. Haensel, C. Kunz, T. Sasaki, and B. Sonntag, Appl. Opt. 7, 301 (1968).

${ }^{9}$ K. Teegarden and G. Baldini, Phys. Rev. 155, 896 (1967).

\title{
TRANSFORMATION TO FAN SPIN STRUCTURE BY EXTERNAL FIELD IN FERROMAGNETIC MnP
}

\author{
Y. Ishikawa \\ Institute for Solid State Physics, University of Tokyo, Tokyo, Japan \\ and \\ T. Komatsubara and E. Hirahara \\ Department of Physics, Tohoku University, Sendai, Japan \\ (Received 14 July 1969)
}

\begin{abstract}
Neutron-diffraction studies have shown that ferromagnetic MnP undergoes a transformation to a periodic fan structure by an external magnetic field. The magnetic moment in the fan is higher than that in the ferromagnetic state. Both the moment and the period of the fan decrease with increasing applied field.
\end{abstract}

Manganese phosphide is ferromagnetic below $291.5^{\circ} \mathrm{K}$, but it transforms to a metamagnetic phase at $50^{\circ} \mathrm{K}^{1}$ In the ferromagnetic state the crystals have an orthorhombic structure $(a>b>c$ for convenience) with the easy magnetization axis along the $c$ direction. The magnetic structure at $4.2{ }^{\circ} \mathrm{K}$ was found by neturon diffraction to be a spiral with propagation vector $0.112 \times 2 \pi / a$ along the $a$ axis. ${ }^{2,3}$ The envelope of the spiral was suggested to be anisotropic. The average magnetic moment per $\mathrm{Mn}$ atom in the $b c$ plane was estimated to be $1.58 \mu_{B}$, which is greater than the ferromagnetic moment of $1.29 \mu_{B}$.

Recent investigation of magnetic and electric properties of single crystals has suggested ${ }^{4}$ that the ferromagnetic structure above $50^{\circ} \mathrm{K}$ transforms to a periodic fan structure when an external magnetic field is applied along the $b$ axis. This paper is concerned with a neutron diffraction study of this transformation. 